\title{
The neutron powder diffractometer DREAM at the ESS
}

Werner Schweika $^{1}$, Mikhail Feygenson ${ }^{2}$, Andreas Houben ${ }^{3}$, Nicolo Violini ${ }^{2}$, Florence Porcher ${ }^{4}$

${ }^{1}$ European Spallation Source ERIC, Lund, Sweden, 2 Jülich Centre of Neutron Science, Forschungszentrum Jülich, Jülich, Germany,

${ }^{3}$ Institute of Inorganic Chemistry, RWTH Aachen University, Aachen, Germany, ${ }^{4}$ Laboratoire Léon Brillouin CEA, Saclay, France

E-mail: werner.schweika@esss.se

The key design parameters for the ESS provide new opportunities in neutron diffraction. The long pulse at 5 MW is most intense and about two orders of magnitude larger than the neutron flux of today's leading pulsed sources. The peak brightness clearly exceeds those of the existing short pulse spallation sources. Tailoring the pulse with fast choppers results in a very flexible time-resolution and yields a unique versatility for measuring either with highest resolution or highest intensity for tiny samples or real-time studies of chemical reactions. This versatility is a characteristic feature of the proposed DREAM powder diffractometer [1], which can ultimately offer a d-resolution of $0.00028 \AA$. Other interesting features with respect to efficiency are the simultaneous use of the thermal and cold ESS moderators, by use of a solid Si bender which will be reflecting the cold neutrons into the incident beam, while transmitting the thermal neutrons. The detectors are based on a new technology using B-10 coated cathodes in inclined geometry [2] covering a large solid angle with position sensitivity appropriate for powder and single crystal diffraction.

The instrument has entered into construction in early 2017. The project scope of the instrument has been set with a budget to deliver a world leading neutron powder diffractometer already with the start of user operation at the ESS in 2023.

The design has been driven by the broad science case received from the European user community. These cases emphasize the needs for neutron diffraction for small or complex samples, in-situ studies of batteries, metal-organic framework structures, and phase-studies with weak signals related to magnetism and superconductivity.

Possible upgrade options cover high-pressure studies with diamond anvil cells and polarized neutrons distinguishing magnetic diffraction or removing the typical large background of hydrogenous materials. With an additional detector for small scattering angles, DREAM will probe multiple length scales within a Q-range from 0.01 to $251 / \AA$, which is sufficient for PDF-studies as well as for small angle scattering of nanoparticles.

Simulating and benchmarking the instrument to world leading instruments demonstrates a far superior performance.

[1] Schweika, W. et al. (2016) J. Physics Conf. Ser. 746012013.

[2] Modzel, G. et al. (2014) NIM A 743 90-95.

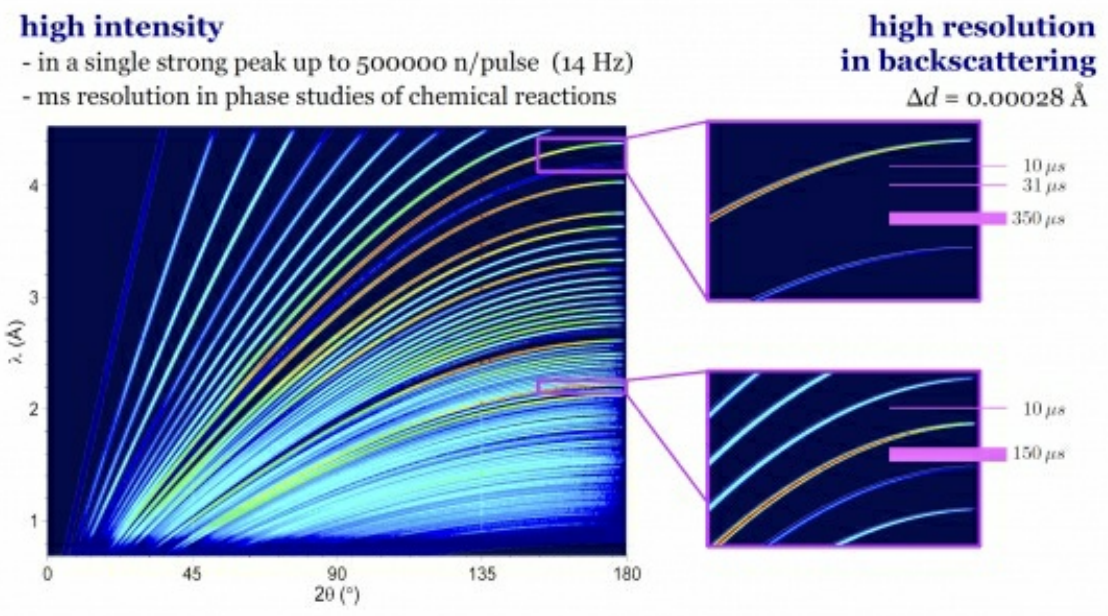

Keywords: Instrumentation, neutron powder diffraction 